\title{
A pilot study to assess the feasibility and acceptability of undertaking acute asthma professional development in three different UK primary care settings
}

\author{
Hilary Pinnock, Gaylor Hoskins, Barbara Smith, Trish Weller, David Price
}

\begin{abstract}
\section{Introduction:}

The General Practice Airways Group's Professional Development programme promotes the evidence-based management of acute asthma in line with current guidelines.

Method:

4 GP practices, 2 Out-of-Hours services and 2 nurse-run Walk-In Centres were recruited to this pilot study. Participants provided organisational data and undertook a critical event analysis of acute attacks. Results were fed back prior to attendance at the GPIAG/NRTC Congress. The audit was repeated after six months. Feasibility and acceptability were assessed with evaluation questionnaires.

Results:

Baseline data from 7 centres (160 attacks) suggested that

management guidelines are not being fully adhered to. For practical reasons only four of the organisations attended the Congress. Organisations successfully formulated their own development plans: one introduced a standard proforma, one commissioned in-service training and two acquired a supply of steroids. Follow-up data was submitted by 5 organisations. (98 attacks) Programme evaluations were generally positive: the audit protocol was practical in all three settings, the feedback was appreciated and the educational intervention was well received by those able to attend.

Conclusion:

This pilot study has provided useful information on which to base the future development of the GPIAG acute asthma professional development programme. The initiative has been well received and innovation has been facilitated. Future projects should consider more flexible, local provision of workshops.
\end{abstract}

\section{Introduction}

Acute asthma is common, ${ }^{1}$ results in 100,000 hospital admissions in England and Wales annually ${ }^{2}$ and is responsible for 1,500 deaths each year. ${ }^{3}$ Studies suggest that management is not optimal ${ }^{4-6}$ when measured against recognised guidelines. ${ }^{7}$ Increasing diversity in the provision of primary care services mean that initiatives to improve the care of acute asthma must include Out of Hours services $(\mathrm{OOH})$, Walk-In Centres (WiC) as well as general practices (GP).

In 1998 the Department of Health, as part of the agenda for improved quality in the NHS, issued a report on the future of continuing professional development in general practice. ${ }^{8}$ It promotes the concept of both practice and personal development plans with the common themes of identifying a need for development, addressing the need and evaluating the progress made. ${ }^{9}$ The Out of Hours Review ${ }^{10}$ expects Co-operatives and Deputising Services to review aspects of professional performance and recommends that plans should include the development of skills related to out-of-hours activity.

Passive educational interventions have been shown to be ineffective in changing professional practice: interactive workshops and active involvement in audit have long been recognised as a tool for promoting change in professional practice. ${ }^{11-13}$ Founded on these concepts the General Practice Airways Group (GPIAG) Professional Development Programme seeks to provide a framework for individuals, practices, Out of Hours services and Walk-In centres wishing to improve their management of acute asthma.

This pilot study aimed to assess the feasibility and acceptability of using the programme across a broad

range of primary care services. The results of this pilot will be used to inform statistical advice for the development of future studies.

\section{Methods}

Ethical approval was obtained for the study from the Tayside Committee on Medical Research Ethics. Recruitment aimed to ensure that the range of primary care services was represented. Nine general practitioners who had previously expressed an interest in GPIAG educational initiatives were approached and four of their practices were recruited to the programme. Four Out of Hours services and three Walk-In centres working in localities known to have identified asthma as a focus for Health Improvement were approached: two of each agreed to participate. Interest in the programme was generally high; those who declined did so because of pressures of time and resources. On enrolment each participant organisation was sent a pack containing information about the programme, a proforma for the baseline audit and educational resources.

\section{The baseline audit}

Organisations were asked to undertake a critical event analysis of acute attacks occurring over a 3 month period to adults and children aged 5yrs and over. An attack was defined as "An acute deterioration of asthma for which the patient seeks urgent medical advice". 6 Participants were advised to use computer databases, discharge letters, out-of-hours service slips, visit requests, prescriptions for courses of oral steroids and nebuliser use to identify all attack episodes in that 3 month period. Once identified, the organisations collected data about management of the attacks from patients' written and computer records. A data sheet, simplified from one used previously ${ }^{6}$ was completed for each episode providing information on the
Hilary Pinnock GPIAG Clinical Research Fellow, University of Aberdeen

\section{Gaylor Hoskins}

University of Dundee

\section{Barbara Smith}

University of Dundee

\section{Trish Weller}

National Respiratory

Training Centre

\section{David Price}

University of Aberdeen

Correspondence to:

Dr Hilary Pinnock

Whitstable Health Centre

Harbour Street

Whitstable, Kent CT5 1BZ

Tel: +44 (0)1227 594400

Fax: +44 (0)1227 771474

E-mail:

hpinnock@gpiag-

asthma.org

Date submitted: $23 / 11 / 02$

Date Accepted: 20/02/03

Prim Care Resp J 2003; 12(1):7-11 


\section{Demographics}

\section{General Practices}

1. Market town practice with 5 GPs and 3 asthma trained nurses

2. Cathedral city with 3 GPs and 1 asthma trained nurse

3. Urban practice with 3 GPs and 1 asthma trained nurse

4. A practice who provided no further data

\section{Out of Hours services}

1. City co-operative of $163 \mathrm{GPs}$

2. Midlands deputising service with 40 doctors

\section{Walk-in centres}

1. City nurse run walk in centre with 11 nurses (3 asthma trained)

2. Town centre nurse run walk in centre with 8 nurses (none asthma trained) assessment of the attack (peak flow, respiratory rate, heart rate, ability to speak) and treatment provided

(bronchodilation, oxygen, steroids) Actions not recorded were assumed not to have been done.

Anonymous data was submitted to the Tayside

Centre for General

Practice for analysis.

Organisations were also asked to complete a questionnaire about the provision they made for the management of acute asthma (availability of peak flow meters, oxygen, soluble steroids, protocol for the management of acute asthma)

\section{The intervention}

- The results of the baseline audit were fed back to participants with comparative data from the other primary care organisations taking part in the programme as well as the standards set by current asthma guidelines.

- The General Practice Airways Group / National Respiratory Training Centre Congress 2001 included a major symposium on the management of acute asthma. A workshop, specifically designed for the participants, facilitated multidisciplinary discussion of practical aspects of care identified by the baseline audit as offering opportunities for development.

- A list of suggested references, resources and practical materials was provided.

- After receiving the audit feedback and attending the Congress, organisations were asked to complete a 'Progress' form designed to encourage them to formulate their own development plans. This invited practices to reflect on their performance, identify aspects of management they wished to improve and make practical plans to overcome barriers and institute change.

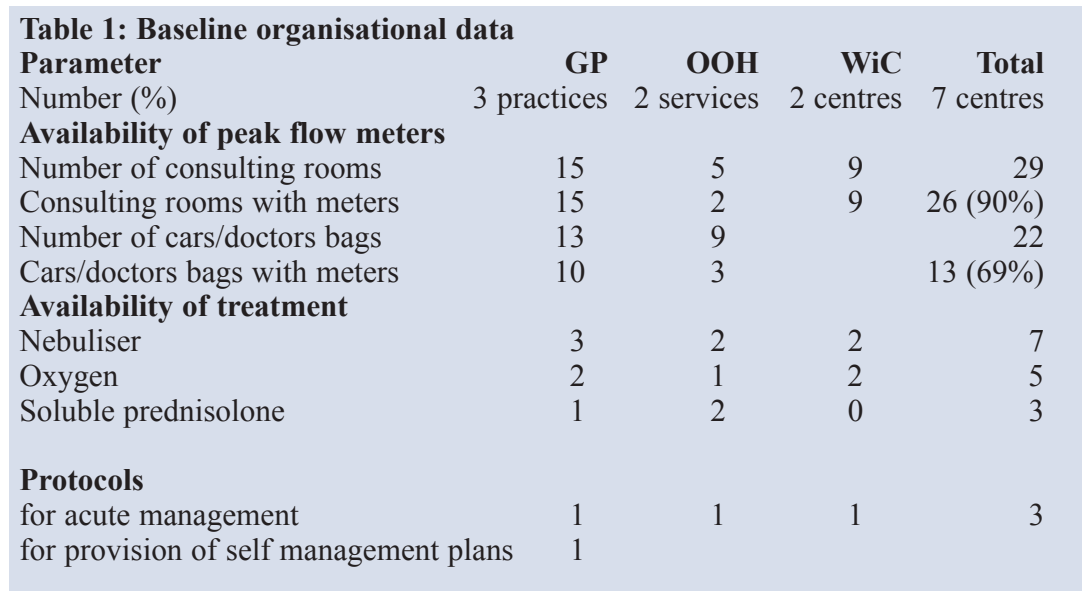

\section{Follow up audit}

The participants repeated the critical event analysis and organisational questionnaire six months after the Congress.

\section{Evaluation}

Participants were asked to complete evaluation questionnaires, designed with the advice of an expert panel, which included questions on all aspects of the programme (feasibility of baseline audit, organisation of project, usefulness of training sessions) using a scales of 1 to 4 with opportunities for free text. The participants were aware that they were taking part in a pilot study and that their comments, criticisms and suggestions would be used to inform the development of future projects.

\section{Quality control}

Independent quality control of the accuracy of the audit data was not possible within the constraints of the budget.

\section{Results}

Four general practices, two Out of Hours services (a GP co-operative and a deputising service) and two nurse-run Walk In centres were recruited. One GP practice subsequently did not contribute to the programme.

\section{Baseline data}

Seven of the organisations completed the questionnaire about their service provision (table 1) and provided audit data on 160 acute episodes. A peak flow was recorded in 77 (48\%) and compared with best/predicted in $33(21 \%)$ of episodes.

Emergency bronchodilation was given to $48(30 \%)$, oxygen was used for 7 (4\%), systemic steroids were given to 36 and prescribed for a further 25 (total 38\%) Fifteen $(9 \%)$ were referred to hospital. Charts 1 and 2 compare management in the different settings.

\section{Intervention}

All organisations received the pack including useful references and resources. The three practices, both the $\mathrm{OOH}$ organisations and both the $\mathrm{WiC}$ received customised feedback. of their baseline audit data. Three GPs attended the Congress (two accompanied by their asthma nurse). The Clinical Governance Facilitator of the $\mathrm{OOH}$ co-operative attended, but the delegates from the deputising service did not attend. The representatives from the two Walk-In Centres were both unable to attend for personal reasons but one centre subsequently arranged a local workshop.

\section{Follow up data}

At the conclusion of the study, five of the organisations (two of the general practices and the Out of Hours organisation who had attended the conference, and both Walk-In Centres) completed the questionnaire about their service provision. During the course of the programme one of the general practices ensured that all their doctors had a peak flow meter in their bag for home visits and two of the organisations arranged to stock soluble prednisolone for immediate administration. The organisations 
Original Research

For personal use only.

Not to be reproduced without the permission of the Primary Care Respiratory Journal

provided audit data about 98 acute episodes. Charts 3 and 4 compare the data provided by these five organisations at the beginning and end of the programme.

\section{Evaluation}

On a scale of 1 to 4 (4 $=$ very satisfactory) the scores for almost all aspects of the programme were between 3 and 4 . No individual components caused particular concerns, though 'timescale' was highlighted as a problem by two of the centres.

The 'Quality Improvement Reports' from a GP practice, a GP co-operative and a Walk In Centre.

\section{Discussion}

The results of this pilot study are both encouraging and instructive.

It is encouraging that the critical event audit appears to be feasible in all three primary care settings. Apart from one practice all the organisations successfully submitted baseline data. They reported no insurmountable difficulties in identifying attacks and the audit proforma was deemed practical and appropriate.

The results of the critical event analysis were similar to previously published data. ${ }^{4-6}$ which provides some reassurance that the methodology of the critical event analysis has been effective. Both these studies concluded that care was suboptimal implying that there is still a need for professional development in the management of acute asthma. One new problem highlighted is the practical issue of providing oral steroids by nurses in Walk-In centres. Current UK regulations do not allow nurses to prescribe steroids, but as a result of this programme one of the Walk-In Centres is developing a 'Patient Group Direction' for the administration of prednisolone (a legal document which allows nonprescribers to provide prescription-only drugs in defined circumstances). ${ }^{14}$

All facets of the programme were rated highly by the participants. There were, however, two practical issues which will need to be addressed in future projects. A national congress is probably not the ideal venue for the educational intervention, though it enabled the provision of a major symposium as well as a workshop. The distances involved and the inflexibility of timing may well have contributed to $50 \%$ non-attendance

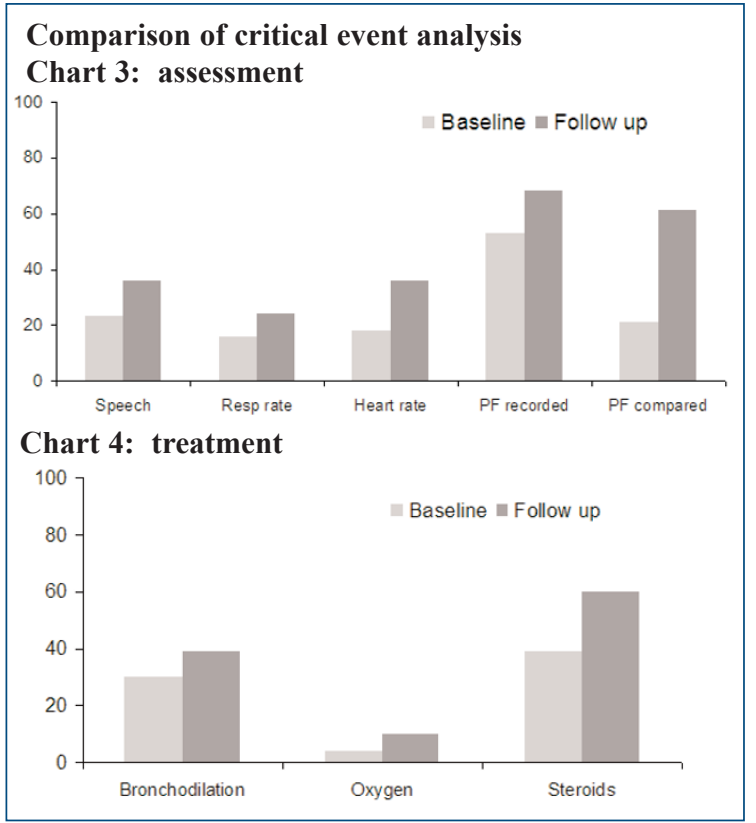

\section{Organisation 3: A GP practice Background}

The practice is in a market town and has five general practitioners caring for 9,500 patients. One of the three practice nurses has responsibility for asthma. We already had a practice guideline for the management of acute asthma based upon BTS recommendations.

\section{Problems identified by the baseline audit}

Coding.Despite being totally computerised not all attacks were coded using the agreed Read code. In order to identify all patients who had had an asthma attack we had to search for patients who had received oral steroids or nebuliser therapy, and identify those who had been admitted to hospital or seen by the deputising service.

Data entry. A lack of assessment data (peak flow, pulse rate and respiratory rate) was evident which made retrospective assessment of the severity of the attacks impossible. While it does not necessarily mean that this information was not obtained by the clinician, it does highlight the need for ensuring that this data is entered.

Secondary care. It was concerning that patients who were seen in secondary care were often not given advice about follow up.

\section{Strategies for change}

Data entry. We are investigating a computer data entry system for acute attacks as an appropriate method of capturing all data.

Secondary care. All correspondence about asthmatics who have been seen in the Accident and Emergency Department, admitted to hospital or who have seen the Deputising Service is passed to our asthma nurse who arranges, either by telephone or by letter, to see them for urgent follow up.

Equipment. The practice now has an emergency bag containing all equipment and medication needed to manage acute asthma: including a nebuliser, oral prednisolone and patient advice leaflets about what to do following an attack. The practice has purchased a high flow oxygen regulator and is in the process of acquiring a pulse oximeter.

\section{Effects of Change}

- Use of oxygen increased from 0 to $20 \%$

- $73 \%$ of patients were followed up at the 6 month audit compared to $59 \%$ at baseline

- Despite the availability of soluble prednisolone it was still prescribed rather than actually given

\section{Conclusion}

We have made progress on improving data entry, the provision of equipment and ensuring follow up though a change of key personnel (two doctors left and were replaced by new partners during the 6 months) may have diluted the impact of the initiatives. It is our intention to repeat this audit process once these steps have been implemented 


\section{Organisation 8: An Out of Hours service}

\section{Context}

We are a GP co-operative providing out of hours care for the patients of $163 \mathrm{GPs}$, the majority of whom work one or two shifts per month. Clinical governance is a government requirement and asthma is one of our Primary Care Trust's priorities.

\section{Problems identified by the baseline audit}

Equipment: Some doctors were not aware of available equipment and many did not know how to use oxygen. Oral steroids were not always available in the visiting doctor's cars.

Clinical records: Clinical records lacked details of assessment: eg: presenting peak flow was never compared with predicted. Advice on follow-up was rarely recorded.

\section{Strategies for change}

The audit results were discussed by the members at the Annual General Meeting

Equipment: A driver was allocated the task of regularly checking equipment in consulting rooms and cars. Asthma guidelines and predicted peak flow charts were displayed in the consulting rooms

Clinical records. We developed a proforma to be attached by our call handlers to the records of any patients presenting with asthma. Designed to prompt the recording of assessment and appropriate action, it was faxed to the patient's GP the next working day to improve communication.

\section{Effects of Change}

- Oral prednisolone is now available for home visits

- We developed a proforma - though it was only used in $17 \%$ of the consultations. (Telephonists forgot to attach it to the records: doctors felt it was time-consuming)

- Consultation records which enabled the severity of the asthma attack to be assessed increased from 5 to $41 \%$

- More patients were given advice about follow up

- Oxygen was still not used. Legal advice about restrictions on the carriage of compressed oxygen mean that oxygen is not available in the cars.

\section{Conclusion}

The asthma development plan coincided with major organisational upheaval. The government's out of hours proposals and being an Exemplar site (piloting links between NHS Direct and out-of-hours organisations) has led to many changes in ways of working and increased paperwork. Many personnel feel overwhelmed and irritated by these changes and saw the asthma development plan as just one more piece of bureaucracy. However, in spite of problems we have demonstrated an improvement in the quality of data recorded and advice given to the patients. We feel that in such a large organisation change occurs gradually and hope that our asthma care will continue to improve as a result of our participation in the project.

\section{Organisation 4: A nurse-led Walk-In Centre}

\section{Background}

The Walk-In Centre is nurse-led, employing 8 permanent $G$ grade staff and 5 bank staff, with no on site medical support. The majority of patients are registered with local general practitioners, however there is a significant number who are not registered with a GP which can cause problems when a medical input is needed.

We agreed to take part in the audit after one of the staff attended an asthma study day. She came back with uncontrolled enthusiasm determined to improve our management of patients attending with acute asthma and shortness of breath. We currently do not have any involvement with long-term management of asthma.

\section{Problems identified by the baseline audit}

Following the baseline audit several issues were raised, Assessment of severity: Although we recorded a peak flow rate not all staff check the patients predicted and calculated the percentage difference.

Management: Oxygen was never used. Patients were nebulised with an air driven compressor.

Organisational: As a nurse run service we were unable to issue the prescription only drug, prednisolone.

Patients requiring systemic steroids were referred back to their GP

\section{Strategies for change}

Staff training: A local study day was arranged with the aim of improving the understanding of the management of acute asthma and increasing confidence when dealing with patients. Although feedback was very positive, unfortunately due to sickness and staffing problems only three Walk-In centre staff attended. Offering the remaining places to local practice nurses and community staff enabled us to improve communication with other providers of primary health care services

Organisational: We are currently drafting a patient group direction to allow the nurses to administer prednisolone.

\section{Effects of Change}

- Presenting peak flows were compared with predicted in $29 \%$ of episodes

- Oxygen is now used to drive the nebuliser.

\section{Conclusion}

Taking part in the audit and the subsequent training day was of great benefit to the centre, despite the fact that not all the staff could attend. One of the consequences of a small team is difficulty in attending training: further study days will be needed. The time taken to write and have Patient Group Directions approved can be laborious, but once in place will be of great benefit to patients. All the staff recognise the need to change our practice in the management of acute asthma. We have started the process and hopefully will be able to deliver improved care in the near future. 
at the Congress. Future programmes will provide local workshops. There were problems, particularly for the larger organisations, in implementing change within the six-month timescale emphasising the need for flexibility.

The 'before and after' design of this small pilot study was not designed to provide statistically valid estimates of change in clinical outcome measures so any apparent improvement should be interpreted with caution. It is however encouraging that individual organisations have instituted changes which ought to result in improved care. Two of the GP practices now have a supply of soluble prednisolone for immediate administration to reduce the delay imposed by providing a prescription. The GP co-operative has designed a proforma for use by members attending an acute episode: a strategy that has been shown to improve performance in an A\&E setting. ${ }^{15}$ The need for local training has been addressed by one of the Walk-In centres. These strategies for change, illustrated by the three quality improvement reports (boxes 2, 3 and 4) are the most striking indicators of the potential of the acute asthma development programme to facilitate change.

\section{Conclusion}

This pilot study has provided useful information on which to base the future development of the GPIAG acute asthma professional development programme. The initiative has been well received: the audit protocols have proved to be practical in all three settings and innovation has been facilitated. Problems with attendance at the Congress and the sixmonth timescale emphasise the need for flexibility in the provision of educational initiatives.

Funding: General Practice Airways Group

Conflicts of interest: None known.

Acknowledgments: The symposium and workshops were part of the GPIAG/NRTC Congress 2001

We wish to thank the participating organisations for the enthusiasm and positive feedback

Capital Road Surgery, 2 Capital Rd, Higher

Openshaw, Manchester, M11 1LA

Carmel Medical Practice, Nunnery Lane, Darlington, Co.Durham, DL3 8SQ

CROYDOC Doctors-on-call, Mayday Hospital,

Thornton Heath, Surrey, CR4 7YE

Croydon Walk-in Centre, 45 High Street, Croydon, CR0 1QD

Healthcall Services Ltd, 152 London Road, Leicester, LE2 1ND

Swindon Walk in Centre, The Health Centre, Carfax Street, Swindon, Wiltshire

Wilton Health Centre, Market Place, Wilton, Salisbury, SP2 0HT

Contributorship: HP initiated the idea for the study and led the development of the protocol, securing of funding, study administration, data analysis,

interpretation of results and writing of the paper. $\mathrm{GH}$ contributed to the development of the protocol, data analysis, interpretation of results and writing of the paper. BS contributed to the data analysis. TW contributed to the educational intervention. DP contributed to the development of the protocol. All authors reviewed the final manuscript.

$\mathrm{HP}$ and GH are study guarantors.

\section{References}

1. Fleming DM, Sunderland R, Cross KW, Ross AM. Declining incidence of episodes of asthma: a study of trends in new episodes presenting to general practitioners in the period 1989-98. Thorax 2000; 55: 657-661.

2. Lung and Asthma Information Agency. Trends in hospital admissions for asthma. LAIA Factsheet 1996; 2.

3. Lung and Asthma Information Agency. Trends in asthma mortality in Great Britain. LAIA Factsheet 1997; 3.

4. Neville RG, Clark RC, Hoskins G, Smith B for GPIAG. National asthma attack audit 1991-2. BMJ 1993;306: 559-62.

5. Neville RG, Hoskins G, Smith B, Clark RA. How general practitioners manage acute asthma attacks. Thorax 1997; 52: 153-156.

6. Pinnock HJ, Johnson A, Young P, Martin N. Are doctors still failing to assess and treat asthma attacks? An audit of the management of acute attacks in a Health District. Respir Med 1999; 93: 397-401. 7. British Thoracic Society. The British Guidelines on Asthma management: 1995 Review and Position Statement. Thorax 1997; 52 (Suppl 1): S1-20.

8. Calman KC. A Review of Continuing Professional Development in General Practice. Department of Health. May 1998.

9. Rughani A. The GP's Guide to Personal Development Plans. Radcliffe Medical Press 2000. 10. Raising Standards for Patients: New partnerships in Out-of-Hours Care. Department of Health. October 2000.

11. Thomson O'Brien MA, Freemantle N, Oxman AD, Wolf F, Davis DA, Herrin J. Continuing education meetings and workshops: effects on professional practice and health care outcomes (Cochrane Review). In: The Cochrane Library, Issue 3, 2002. Oxford: Update Software.

12. Thomson O'Brien MA, Oxman AD, Davis DA, Haynes RB, Freemantle N, Harvey EL. Audit and feedback versus alternative strategies: effects on professional practice and health care outcomes (Cochrane Review). In: The Cochrane Library, Issue 3, 2002. Oxford: Update Software.

13. Cantillon P, Jones R. Does continuing medical education in general practice make a difference. $B M J$ 1999; 318: 1276-9.

14. Department of Health 1997 Supply \&

Administration of Medicines under Group

Protocol[Crown Report].

15. Robinson S,Harrison B, Lambert M. Effect of a preprinted form on the management of acute asthma in an accident and emergency department. J Accid Emerg Med 1996; 13: 93-7. 